Carnets de géographes

GÉOGRAPHES

\title{
Produire la ville en Asie du Sud-Est. Les stratégies socio-spatiales des acteurs immobiliers à Phnom Penh, Cambodge
}

\section{Gabriel Fauveaud}

\section{CpenEdition}

\section{Journals}

Édition électronique

URL : http://journals.openedition.org/cdg/903

DOI : $10.4000 /$ cdg.903

ISSN : 2107-7266

Éditeur

UMR 245 - CESSMA

Référence électronique

Gabriel Fauveaud, « Produire la ville en Asie du Sud-Est. Les stratégies socio-spatiales des acteurs immobiliers à Phnom Penh, Cambodge », Carnets de géographes [En ligne], 6 | 2013, mis en ligne le 01 septembre 2013, consulté le 24 septembre 2020. URL : http://journals.openedition.org/cdg/903 ; DOI https://doi.org/10.4000/cdg.903

Ce document a été généré automatiquement le 24 septembre 2020.

\section{(i) $९$

La revue Carnets de géographes est mise à disposition selon les termes de la Licence Creative Commons Attribution - Pas d'Utilisation Commerciale - Pas de Modification 4.0 International. 


\title{
Produire la ville en Asie du Sud-Est. Les stratégies socio-spatiales des acteurs immobiliers à Phnom Penh, Cambodge
}

\author{
Gabriel Fauveaud
}

1 Cette thèse de doctorat en géographie / études urbaines s'intéresse au rôle des acteurs citadins dans la réorganisation des dynamiques immobilières de Phnom Penh. Petite métropole secondaire sud-est asiatique peuplée d'environ 1700000 habitants, Phnom Penh se transforme rapidement depuis le milieu des années 1990 au rythme de l'accélération d'investissements immobiliers locaux et étrangers. L'évolution du cadre bâti en périphérie comme dans la ville-centre évoque un effet de rattrapage de l'urbanisation de la capitale cambodgienne après le véritable « urbicide » subi pendant le régime Khmer rouge entre 1975 et 1979 : la ville est vidée de ses habitants pendant près de quatre ans, presque la moitié de la population urbaine décède et une partie importante des bâtiments et infrastructures sont détruits ou endommagés.

2 La reconstruction contemporaine de la capitale cambodgienne va de pair avec la réorganisation rapide des marchés immobiliers. Ce secteur économique bénéficie de la redirection d'une partie importante des gains de la croissance économique dont ont principalement bénéficiée des Cambodgiens issus de la diaspora, certains groupes citadins (principalement des fonctionnaires et des marchands) et des ménages provinciaux enrichis dans le commerce de matières premières. La réorganisation des marchés immobiliers de Phnom Penh se caractérise donc par la coexistence de pratiques immobilières à la fois professionnelles et amateurs et par l'intervention concomitante d'acteurs spécialisés dans le secteur immobilier et d'acteurs individuels et familiaux qui tirent parti de la spéculation foncière.

3 Au cours de cette recherche, nous avons choisi de mener de front deux principales approches théoriques. Tout d'abord, nous avons considéré la «production d'espaces urbains » de la capitale cambodgienne à la fois dans sa dimension verticale - par 
l'examen de la filière de production de biens immobiliers - et dans sa dimension horizontale, de la production de biens immobiliers à la constitution de territoires citadins et d'espaces de vie.

4 Ensuite, nous avons construit une approche socio-spatiale, qui considère l'immobilier comme un champ socio-économique particulier, investit par des acteurs mobilisant différentes formes de capitaux (aussi bien économiques que sociaux, culturels, symboliques et spatiaux) pour assoir leurs stratégies, maintenir ou faire évoluer leur position dans le champ. Cette recherche s'est finalement organisée autour de deux questions centrales, qui abordent deux facettes de la production d'espaces urbains :

Comment la production de biens immobiliers participe-t-elle à la construction et à la transformation des territoires urbains de Phnom Penh?

D'un autre côté, dans quelle mesure les dynamiques sociales, économiques et territoriales citadines déterminent-elles l'organisation de la production immobilière?

7 Notre travail de terrain, mené sur 26 mois, s'est caractérisé par un travail d'observation et de décorticage de multiples opérations immobilières. Afin d'embrasser l'ensemble du processus de production d'espaces urbains, nous avons par ailleurs considéré un large spectre d'acteurs immobiliers. Un peu plus de 500 entretiens semi-directifs, à la fois quantitatifs et qualitatifs, ont été menés auprès de ménages habitants des espaces péricentraux et périurbains de Phnom Penh, pour mieux comprendre le caractère collectif de l'activité immobilière, le rôle des parcours et stratégies résidentiels dans l'organisation du commerce et de la production de logements et la manière dont les individus mobilisent et utilisent, au niveau local, les ressources immobilières. Nous avons parallèlement réalisé des entretiens auprès de grandes familles aisées de la capitale cambodgienne. Enfin, une attention particulière a été accordée aux acteurs institutionnels, aussi bien au niveau de la Municipalité de Phnom Penh et de certains ministères qu'au sein des espaces locaux en périphérie de la ville.

Finalement, cette recherche a permis d'identifier certains éléments fondamentaux qui caractérisent la réorganisation des marchés immobiliers de la capitale cambodgienne. Les marchés immobiliers représentent bien un champ socio-économique particulier déterminé par une organisation hiérarchique de ses acteurs, qui sollicitent différentes formes de capitaux pour assoir leurs stratégies et leur position de domination à l'intérieur du champ.

9 Les grands investissements importants représentent la partie visible de négociations politico-économiques opaques prises au sommet de la hiérarchie institutionnelle. Les projets immobiliers les plus importants sont les moteurs de la ville-projet et de la villespectacle, qui montrent que Phnom Penh est entrée de plain-pied dans la compétition inter-urbaine régionale. Mais la capitale cambodgienne représente aussi un prisme déformant des processus de transferts de modes de production allochtones d'espaces urbains, qui tempèrent la présupposée uniformisation des espaces urbains aux échelles régionales et internationales. Bon nombre de projets immobiliers représentent en ce sens une synthèse de formes architecturales d'influences cambodgienne, sud-est asiatique et internationale adaptées au caractère multiple des aspirations citadines locales.

10 À un autre niveau d'analyse, la production contemporaine d'espaces résidentiels représente une forme d'inscription spatiale de la nouvelle place sociale et économique qu'occupent certains groupes sociaux intermédiaires et aisés dans la cité. Ces derniers 
sont au cœur de la reformation de groupes d'intérêts citadins, qui savent tirer parti, parfois dans l'ombre des quelques grandes familles cambodgiennes et sino-khmères situées au sommet de la hiérarchie institutionnelle, des potentialités économiques de l'urbanisation du capital immobilier.

Pour les habitants enfin, la participation au marché du logement est conditionnée par le déploiement de stratégies et tactiques particulières. Au sein d'un marché résidentiel particulièrement opaque, la recherche d'informations spatiales et l'acquisition de savoirs territoriaux conditionnent le positionnement des acteurs vis-à-vis des marchés immobiliers de Phnom Penh. Si la sollicitation des réseaux filiaux et parentélaires représente le moteur de l'élaboration des stratégies immobilières, bien d'autres ressources sont mobilisées. Les expériences résidentielles et spatiales permettent à certains acteurs de tirer leur épingle du jeu et parfois de contourner l'organisation exclusive du champ immobilier. La captation de connaissances spatiales contenues au sein de lieux producteurs, diffuseurs et relais d'informations permet par ailleurs aux acteurs immobiliers de maintenir leur position de domination au sein du champ immobilier ou, au contraire, de se jouer des relations domination et de l'organisation hiérarchique du champ.

La production d'espaces résidentiels à Phnom Penh au prisme des stratégies d'acteurs

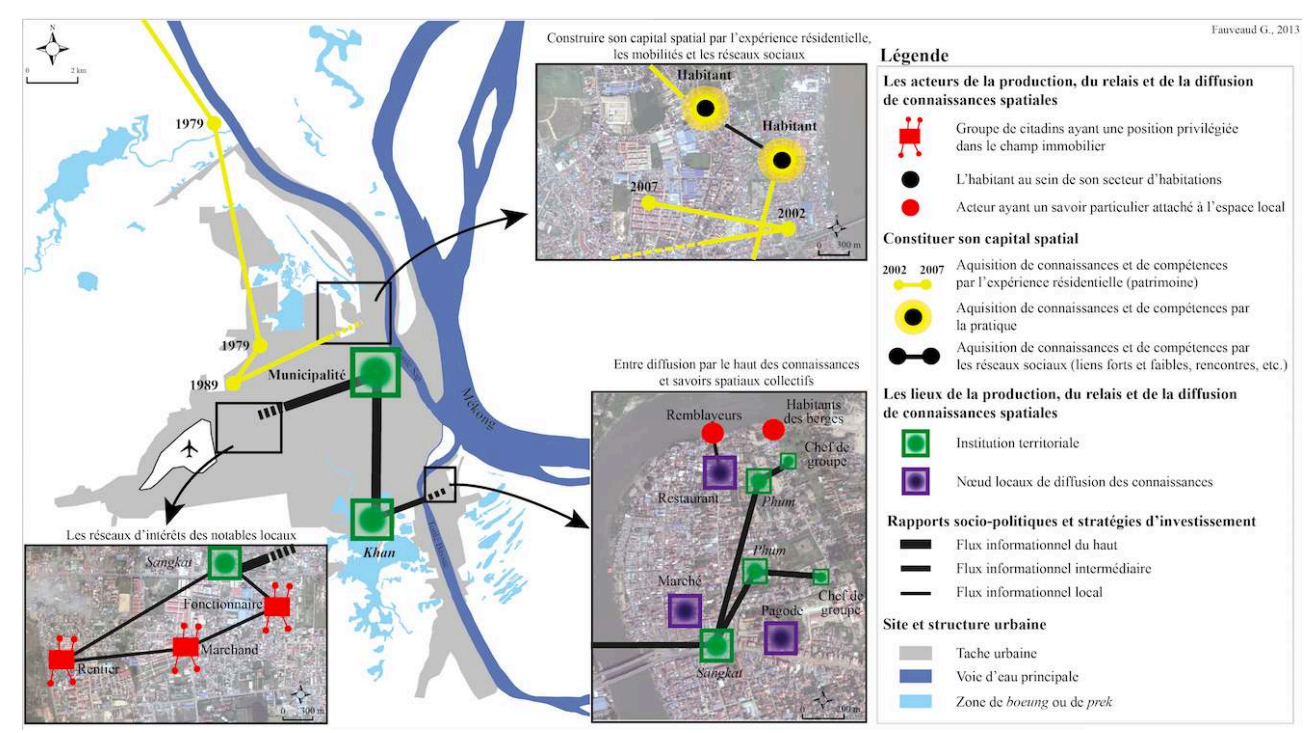

Finalement, ce travail de recherche souligne la nécessité de considérer la production immobilière, et particulièrement la production de logements, au croisement des dynamiques sociales et territoriales, qui participent de la construction d'espaces citadins singuliers au Cambodge et, plus largement, en Asie du Sud-Est. Au sein de ce cadre d'analyse, les lieux, l'espace et les territoires sont bien plus que les supports de l'urbanisation: ils sont des ressources essentielles qui structurent les stratégies des acteurs urbains.

\section{Discipline}

Géographie / Aménagement - urbanisme

\section{Directeur}

Thierry Sanjuan 
Université

Université de Paris 1 Panthéon-Sorbonne

Membres du jury de thèse, soutenue le 13 février 2013

Mme Martine BERGER, Professeur émérite, Université de Paris 1 Panthéon-

Sorbonne (Président du Jury)

M. Rodolphe DE KONINCK, Professeur, Université de Montréal (Rapporteur)

M. Charles GOLDBLUM, Professeur émérite, Université de Paris 8 Vincennes-SaintDenis (Rapporteur)

M. Éric HUYBRECHTS, Architecte - urbaniste, Institut d'aménagement et d'urbanisme de la région île-de-France

M. Thierry SANJUAN, Professeur, Université de Paris 1 Panthéon-

Sorbonne (Directeur de thèse)

Situation professionnelle à l'issue de la thèse (2013 - 2014)

Post-doctorant au Centre d'études et de recherches internationales de l'Université de Montréal (Cérium). Post-doctorant à l'Observatoire Ivanhoé Cambridge du développement urbain et immobilier (OIC), Institut d'urbanisme de l'Université de Montréal

\section{Courriel de l'auteur}

Gabriel.fauveaud[at]gmail.com

\section{INDEX}

Thèmes : Carnets de soutenances

Mots-clés : Immobilier, ville, Cambodge 